\title{
IoT-based systems for improving older adults' wellbeing: a systematic review
}

\author{
Imran Khan, Ana Carla Amaro and Lídia Oliveira \\ DeCA, Universidade de Aveiro \\ Digimedia - Digital Media and Interaction (CIC.Digital \\ Campus de Santiago, 3810-193, Aveiro, Portugal \\ imran10111@ua.pt,. aamaro@ua.pt, lidia@ua.pt
}

\begin{abstract}
Physical, social and psychological wellbeing is one of the most critical issues among older adults' population. Several Internet of things (IoT)-based systems have been developed, in the last 8 years, for improving older adults' wellbeing and support an autonomous and healthy lifestyle. This paper presents the results of a systematic review, aimed to understand how IoT and IoTbased systems have been designed and used, for the past 8 years, for improving the wellbeing of older adults. A total of 173 studies 2 , published between 2010 to 2018, were selected for review. Results show that the selected studies are mainly related to healthcare and social networking applications, and involve sensors, cloud computing, fog computing and advanced communication networks.
\end{abstract}

Keywords - older adults; IoT; ICT; wellbeing; healthcare.

\section{INTRODUCTION}

In western societies, older adults' population is becoming isolated, due to smaller social circles and to physical and psychological health issues [1].

Typically, as age increases, the need for support and medical assistance also increases. Nowadays, technology developments brought revolutionary digital applications and platforms, which facilitate access to physical and psychological healthcare, aimed, in particular, at older adults' population.

Internet of Things (IoT), for example, has the potential to play a very significant role in designing an appropriate healthcare system for dependent elderly people [2].

IoT is shifting traditional healthcare paradigm from a doctorcentred environment to a patient's centric healthcare environment [1], because the characteristics of IoT applications are making it possible to provide older adults' remote monitoring, both from short and long distances. Furthermore, IoT components have the ability to cooperate with new digital technologies and networking facilities that enhance the IoT effectiveness and efficiency in social networking [3].

The IoT technology is an affordable solution, with less power consumption and easy to operate IoT applications [4]. However, the fact that social interactions change according to age, mental and physical health condition, must be considered while designing, developing and commercializing IoT-based healthcare applications, especially when older adults are a targeted group [5].

Although several IoT-based healthcare technologies and applications are being developed for supporting older adults' wellbeing, their usage rate by older adults is very low, due to low levels of Information and Communication Technologies (ICT) literacy and the conservative perception of older adults about ICT $[6,7]$.

In this way, it seems crucial to understand how IoT-based systems can be designed, in order to improve older adults' physical, psychological and social wellbeing and so that they can be easily used by this target audience, considering their specificities.

Therefore, an extensive systematic review, from 2010 to 2018, was conducted, searching for IoT-based systems and studies, which had older adults as the target audience, thus highlighting their issues and specificities, and approaching the novel solutions that are contributing to, and promoting, older adults' physical, psychological and social wellbeing.

\section{BACKGROUND}

In the last few decades, the population of the developed and developing countries are getting older, due to the increase in life quality and medical facilities. In fact, it is expected that $50 \%$ of the European population will be 60 or older by 2040 [8].

Life expectance increasement raises various political, societal, economic and health challenges, at psychological, physical and social level $[9,10]$. As age increases, people become potentially more fragile, since society associates age with physical and psychological impairment [11]. Some factors increase the intensity of fragilities, such as depression, chronic diseases, loneliness, and a smaller social circle [12,13].

If there is no way for older adults to overcome their health challenges, the likelihood of physical and psychological consequences increases [14]. The fact is that many countries' government, the private sector, and institutes are neglecting older adults' issues and there is no serious visible effort to address and resolve older adults' issues [14].

Portugal is one of the countries where population ageing is a major societal challenge. The Portuguese population is declining, since birthrate has been lower than the death rate [4]. According to the Portuguese National Institute of Statistics [15], $29.1 \%$ of the Portuguese population is 60 or older, $3.7 \%$ of which is living in villages, with loneliness and social isolation. In fact, many older adults live alone, since their children and grandchildren migrated to the big cities, in search of jobs, education, and a better quality of life. 
In general, social isolation and age factor leads to psychological and physical health issues, such as depression, anxiety and even heart attacks. Actually, loneliness and social isolation have the same effects on health as smoking on the heart, increasing the blood pressure and weight that causes the heart attack [13].

With the development of novel emerging technologies like IoT, people's life has been improved. The IoT is an innovative and growing concept, in which everyday objects are digitally augmented and linked with computers, mobiles, vehicles and other objects $[5,16]$.

Due to connectivity, IoT is helping to support older adults' physical, psychological and social issues and needs, through remote monitoring healthcare applications, such as Zigbee wireless technologies, which are designed for fall detection [17]. Another IoT application is Active Assisted Living (AAL), which is capable of providing video camera surveillance, in order to monitor older adults' indoor activities [18].

Moreover, Body Sensors Network (BSN) is also a novel and emerging application in IoT technologies, intended for measuring and analyzing different functions of the human body, such as blood pressure, oxygen in the blood, heartbeat rate, and body temperature [19].

In this way, seems possible to state that IoT has an important contribution in the promotion of older adults' physical wellbeing, in increasing the social interactions and expanding social circles, motivating them to participate in the social events. The systematic review hereby described aimed to identify how these contributions have been happening.

\section{METHODS}

\section{A. Research question}

The current systematic literature review seeks an answer to the following question:

How IoT and IoT-based systems have been designed and used, for the past 8 years, for improving the physical psychological and/or social wellbeing of older adults?

\section{B. Goals of the review}

a) Identify and characterize the most significant research on the role of IoT in supporting and promoting the social, physical and/or psychological wellbeing of the elderly, undertaken in the last 8 years;

b) Identify the main technological and design features that proved to be effective in improving either social, physical and/or psychological wellbeing of the elderly.

\section{Search strategies and procedures}

The search was carried out in the Scopus database, using three different search queries. The main reason behind the strategy of using three instead of just one query was the way Scopus works. Often, the Scopus database does not return appropriate results in response to long queries or returns an error as a result of the use of long lists of keywords and sub-keywords.

\section{Inclusion and exclusion criteria}

The documents selected for the systematic review cumulatively met the following inclusion criteria:

a) Have been published between 2010 and 2018;

b) Address IoT from the perspective of older adults' wellbeing;

c) Consider as target population people aged 60 years old or more;

d) Sufficiently describe the design or usage of IoT solutions or IoT-based systems developed to promote older adults' wellbeing;

e) Present a sound methodological approach;

f) Sufficiently describe results, highlighting the importance of the system for older adults' wellbeing, either at physical, psychological or social levels.

Were excluded from the review all the documents that did meet the inclusion criteria.

\section{E. Data extraction and analysis}

The inclusion and exclusion criteria were applied by the researcher, based on the reading of the titles and abstracts of the papers. Only when the titles and abstracts did not allow an unambiguous evaluation, the papers were fully read. In cases of doubt, the opinion of a second investigator was requested.

In order to synthesize the data to be extracted from the selected papers (after applying the inclusion and exclusion criteria), an e-form was designed, which was prepared to compile the information related with the main categories of the analysis, namely: theoretical argumentation, targeted population, methodological approaches, functionalities of the IoT-based systems and final outcomes of the studies.

Considering the heterogeneity of the selected studies, namely regarding methodological approaches and results, the use of statistic synthesis to analyze and present the results was not possible. Instead, the narrative synthesis was used, tabulating the results for their successive synthesis.

In this way, and through this method, a synopsis and a highlevel comparison of selected studies and their characteristics was made, allowing us to understand the contribution of each study to the global synthesis and thus provide an answer to the question that underlies this review, unveiling the features of IoTbased systems that have been developed for improving the physical, psychological and/or social wellbeing of older adults.

\section{RESULTS}

The three search queries ${ }^{1}$ used on Scopus resulted in a list of 283 publications, from which $173^{2}$ publications have been included in the review and the remaining 106 excluded, due to not fulfilling inclusion criteria.

From the set of publications included in the review, $69^{2}$ describe studies on IoT and the physical wellbeing of older adults, involving, in particular, real-time monitoring, fall detection, physical data monitoring, and stroke detection. The remaining 
$104^{2}$ publications addressed IoT and the psychological and social wellbeing of the older adults.

\section{A. Geographical origin of the reviewed studies}

The reviewed studies were conducted in 42 countries, but most of the studies come from India $(n=19)$ and China $(n=10)$, as shown in Figure 1.

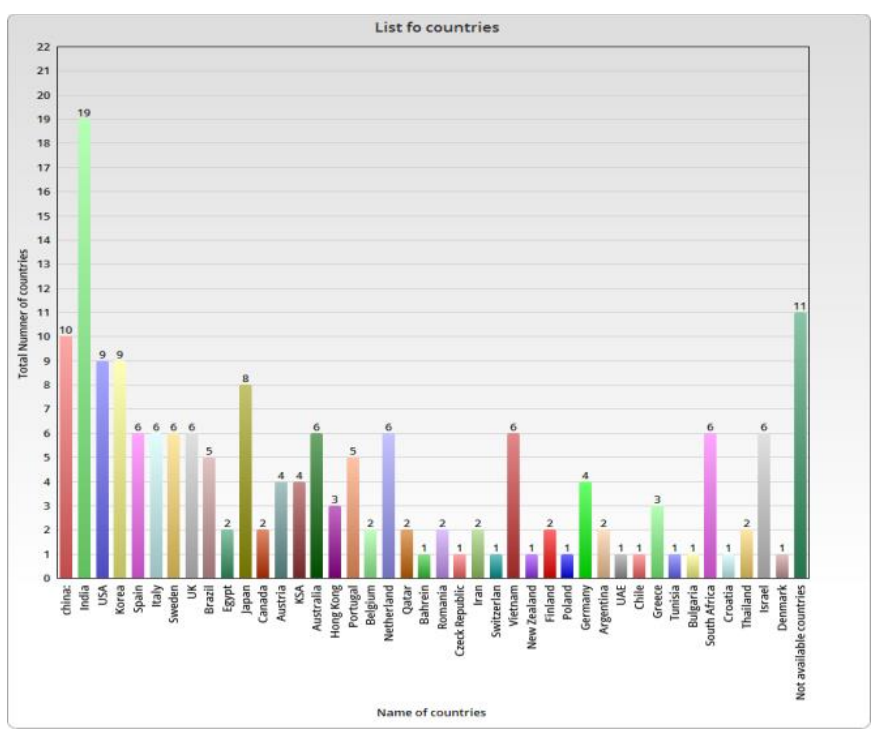

Figure 1. List of countries.

\section{B. Different Methodological approaches used in reviewed studies}

As showed in Figure 2, the reviewed studies used different methodological approaches to address the improvement of older adult's wellbeing through IoT. However, most of the studies were based on qualitative methods.

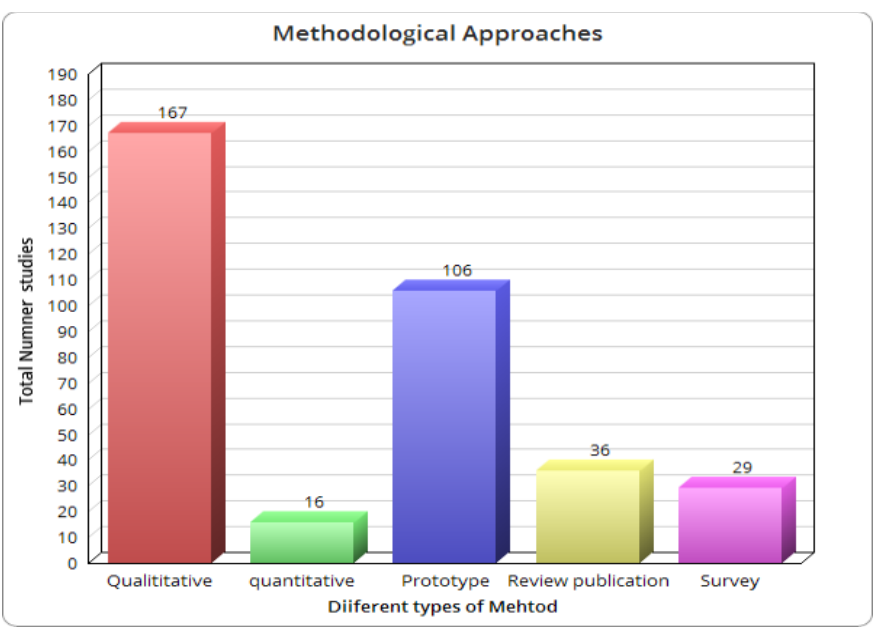

Figure 2. Methodological Approachs.

\section{Main technologies of the reviewed systems}

In 109 of the selected studies ${ }^{2}$, prototype platforms for monitoring older adults were described. These prototype platforms are connected with smart devices, sensors (fall detection), web-based applications, (social, video conferencing, chat rooms, email, and search), and camera for video surveillance and safety of the older adults.

From the analyzed studies it was possible to identify a set of 5 fundamental technologies, used in the development of IoTbased systems to support older adults' wellbeing, namely:

1) Safety technologies: Seven of the reviewed studies focus on data privacy and secure authentication. In the field of older adults' healthcare and monitoring, security and privacy are multifaceted issues [20], as demonstrated by the study $111^{2}$, which presents a survey on the security and services of IoTbased applications and systems. Besides, data security is considered the most important privacy issue concerning IoTbased healthcare systems, as showed by study $1071^{2}$. In fact, the reviewed studies propose very interesting solutions at this level. Study $44^{2}$, for example, describes a security and authentication scheme, called "naked environment", which recognizes older adult identity and data, through automatic biometric authentication. Study $941^{2}$, on its turn, proposes an IoT-based approach called "6LoWPAN AND RFID /NFC", which has great strength and reliability in providing communication, physical monitoring, and control. RFID/NFC technologies provide a secure platform for communication with IoT-based medical devices, based on SIM card verification, encoding and signing in, ensuring older adults' privacy. Furthermore, study $111^{2}$ is concerned with how to secure the sensors data in users' perspective, proposing "Named Data Networking", whose basic function is to carry the digital information such as voice, video and digital text. It also contains various nodes that are interlinked with numerous communication channels. Finally, study $421^{2}$ presents a very novel voice recognition scheme that helps doctors or caregivers to estimate the situation of older adults and patients, by observing their voices, mood, and background noise.

2)Intelligent networks: In IoT-based healthcare systems, data collection is done through bio optic smart sensors, embedded in different intelligent devices and networks that gather real-time monitoring data from older adults. Those sensors can sense and monitor electroencephalograph (EEG), electrocardiogram (ECG), blood pressure, heart bit rate, glucose, virus and so on. Wireless Sensors Network (WSN) is a comprehensive tool for the intelligent network for IoT healthcare systems or platforms. The WSN technologies are equipped with IP addresses, making them capable of providing a solution for smart objects and IoTbased devices. Unequivocally identified by an IP address, any sensor-augmented object, computers, RFID tags, smart devices or phones, are able to actively connect with networks and

funds, and by the European Regional Development Fund, framed in the Operational Programme for Competitiveness and Internationalisation COMPETE 2020, under the new partnership agreement PT2020.
This research was carried out within the scope of LOCUS project. LOCUS is co-funded by FCT - Foundation for Science and Technology, through national 
efficiently collaborate to complete different tasks. The data or information collected from intelligent networks is stored in the cloud, giving rise to the concept of "Cloud Computing". So, through cloud computing, it is possible to manage the data and distribute it remotely. In all the 173 studies $^{2}$ reviewed, smart sensors and intelligent networks were used to improve the productivity of the proposed healthcare systems.

3) Could computing: In addition to ensuring the safety of older adults' physical data, IoT can provide a virtual storing platform, called cloud computing, throught which authorized personnel, such as doctors, caregivers and relatives, can access older adults' physical and medical data. As stated before, cloud computing is a very comprehensive and vital technology, that is capable of storing data in data centres and via hosted servers, interconnected with each other via internet services. Cloud computing is playing a very vital role in healthcare systems, since, through Cloud-IoT, it is possible to provide sensing services in the context of sensor data accessibility and usage. In our research results, studies 42 and 46 are specifically based on cloud computing technologies. These two studies propose Health monitoring system sensors (HM-SS) oriented sensing services scenarios, demonstrating that cloud computing services improve data quality and accessibility and that, through cloud computing, it is now possible to keep an eye on older adults' health and access their physical and medical data, providing them with relevant aid related to their physical, psychological and social wellbeing.

4) Big Data: In this systematic review, three studies $(7,28$, and 62$)^{2}$ focus on the concept of Big Data. Big Data performs by using machine learning tool, which is based on the science of developing computers to perform tasks without being explicitly programmed. In fact, the machine learning tool is the technology behind, for example, intelligent cars and voice recognition and it works by enhancing performance standard using data or previous experience [21]. After the emergence of cloud computing technology into IoT technology, there was the need of a tool to efficiently and quickly manage huge amounts of data, coming from different IoT-based health monitoring services or platforms and big data come as a solution.

5) Fog computing: Two of the reviewed studies $(5,128)^{2}$ were based on fog computing concept. Fog computing is an architectural approach that increases the radius of cloud and big data computing by using edge devices, which are responsible for carrying out a substantial amount of computational tasks that were before executed by servers in a data-centre, thus reducing systems' response time. Within the scope of healthcare IoT-based systems for older adults, it is very important to respond to an emergency basis especially in a critical situation. In this way, fog computing can support the improvement of older adults' physical wellbeing, enabling IoTbased healthcare and monitoring in real time.

D. Main technological paradigms for improving older adults' wellbeing

The Active Assisted Living (AAL) is a novel and emerging multi-faceted area at the interlink between communication and information medical research, technologies and sociological science, which allows setting up a personal healthcare system. The AAL platforms are the infrastructure of computers that is contained on software and hardware. The computing architecture allows to program applications and manages their activities. According to the comprehensive definition by Ducatel et al. [22], AAL is "A vision of people surrounded by intelligent intuitive interface that are embedded in all kinds of objects and environment that is capable of recognizing and responds to the presence of different individuals in an invisible way". The analyzed AAL studies $\left(46,59,77,85,93\right.$ and $\left.112^{2}\right)$ are based on an architecture that is capable of integrating high quality healthcare, medical and comfort facilities, to gain the interoperability, usability and considering older adults' capacities to handle situations.

The AAL concept promises better life quality environments for the older adults and for people with chronic diseases, social isolation and in illness retrieval status by designing and developing innovative technologies and services. AAL provides more security, care, and interaction that helps to improve the physical, psychological and social wellbeing of older adults.

\section{E. Different approaches identified in selected suites}

1) Information and Communication Technologies (ICT) systems: Selected studies ${ }^{2}$ ranging from 122 to 173 are based on ICT concept, from which a total of 12 studies $^{2}(123,127,132$, $137,138,140,149,152,160,164,165$ and 166) have ICT in their titles. These studies results demonstrate that ICT, computers and internet use, can promote the overcoming of social interaction problems between older adults [23]. Despite the numerous obstacles that hinder the use of technology by older adults, including less digital literacy, research has been showing that an increasing number of older adults are now exposed and using new technologies in their daily life activities [24]. ICT in general, but mainly the internet, social media and email, are offering older adults new ways to communicate with relatives, friends and with those who have a common interest, and are providing direct access to an extensive variety of information, data, and resources.

2) Medical and Healthcare: AAL has been playing a very vital role in enhancing older adults' medical and healthcare. Throught AAL, it is possible for non-medical staff to use devices which were, in the past, only available to medical staff and doctors. Nowadays, due to AAL technology, it is possible for relatives, for example, to use medical devices for older adults' health improvement and monitoring as, for instance, wearable devices and remote control monitory applications. In this review, eighteen studies $(01,05,09,11,13,25,28,30,34$, $36,42,53,81,91,92,113)^{2}$ are based on AAL for emergency response and as a fall detection solution, and two studies (17, $32)^{2}$ indicated that the AAL system provides video surveillance to older adults. Furthermore, AAL technologies provide support in monitoring older adults' activities of daily living (ADL) (see study 15$)^{2}$, through producing reminders (see study 25) ${ }^{2}$ and by allowing older adults to get connected with medical staff or caregivers. 


\section{DISCUSSION}

This systematic review aimed to address the potential of IoT for increasing the physical, psychological and social wellbeing of older adults and preventing the social isolation. The final outcomes of this systematic review provide qualitative and quantitative evidence of the positive role of IoT-based platforms in promoting older adults' physical, psychological and social wellbeing. The systematic review provided an overview of the strategies, rules, and reliable practices.

Almost all of the reviewed studies assessed the effect of IoT use on wellbeing, at physical, psychological and/or social (social support, social exclusion and social connectedness) levels. These results indicate that older adults' physical wellbeing is a multifaceted concept. Although there are clear signs that IoTbased systems have a direct effect on improving physical wellbeing, their impact on the perception of physical wellbeing by older adults remains partly unidentified. In this way, more research is needed in order to open new horizons that help to unlock the relationship between IoT and physical wellbeing improvement.

This paper explores the various technologies, systems, and technological paradigms found in the undertaken systematic review and how these efforts can be extended towards emerging novel technologies for the IoT concept.

Up to the present time, it seems that physical, psychological and social wellbeing are, in fact, the core areas that interest researchers on pervasive and ubiquitous technologies. In the healthcare domain, a wide range of hybrid healthcare scenarios already exist, based on an ecosystem of connected objects, which improve older adults' physical conditions.

These systems use IoT-based devices for monitoring physical activities, intended to, for example, fall detection (see studies $^{2}$ 17, 28, 36, 53 and 90), strokes prevention (see study ${ }^{2}$ 25) and detection of early symptoms (see studies ${ }^{2}$ 80, 112 and155). They also use Big Data and Could Computing to store and manage data (see studies ${ }^{2}, 32,43,46$ and 127), that doctors, family members and authorized people can access and take appropriate actions.

Some of the selected studies have extensive components that help to promote the wellbeing by developing prototype smart homes (see studies ${ }^{2} 18$ and 69) that have video and video call surveillances (see studies ${ }^{2} 17$ and 32). Moreover, there are three studies (see studies ${ }^{2}, 92$ and 153) that provide home telehealth monitoring services.

In the last eight years, many novel technologies emerged into the IoT concept, for example, Arduino (see study ${ }^{2} 30$ ), semantic (see studies 231 and 98), fusion approach (see study ${ }^{2}$ 50), Ubi: Liven (see study ${ }^{2} 54$ ), IReHMo (see study ${ }^{2} 73$ ), Ubi Held (see study $^{2}$ 90), and Neuro-Fuzzy (see study ${ }^{2}$ 97). These technologies can increase the healthcare support services and enhance older adults' wellbeing.

The mix of IoT and ICT (see studies ${ }^{2}$ 123, 127, 132, 137, $138,140,149,152,160,164,165$ and 166) seems to have the ability to improve the social and psychological wellbeing of older adults, by helping to reduce social isolation, to create new contacts or networks (see studies ${ }^{2}$ 135, 138, 147, 152, 157 and
173), to established social support and social connectedness effectively. In this context, IoT's social networking seems capable to reduce the social isolation (see studies ${ }^{2} 123,134$, 137), loneliness, depression, anxiety and the intensity of these symptoms.

Likewise, the IoT social environment (ICT-based) can engage older adults in social relations and communication, thereby improving the social and psychological wellbeing of older adults.

\section{CONCLUSION}

In conclusion, this systematic review showed that, in the last 8 years, more well-designed studies have been conducted on the effects of IoT technology on physical, psychological and social wellbeing of elderly people.

In fact, this study demonstrates that IoT is a comprehensive and extensively promising tool for improving older adults' wellbeing. Moreover, the review's results suggest that rapid development of ICT and Social Internet of Things (SIoT) can be helpful to promote older adults' psychological, physical and social wellbeing.

Also, the results of this systematic review can assist the conceptualization and development of advanced and effective IoT-based designs and technologies to promote the physical, psychological and social wellbeing of older adults.

\section{REFERENCES}

[1] de Jong Gierveld, J., \& Tesch-Römer, C. (2012). Loneliness in old age in Eastern and Western European societies: Theoretical perspectives. European Journal of Ageing, 9(4), 285-295. https://doi.org/10.1007/s10433-012-0248-2

[2] Uribe, J. A., Duitama, J. F., \& Gaviria Gómez, N. (2011). Personalized message emission in a mobile application for supporting therapeutic adherence. 2011 IEEE 13th International Conference on E-Health Networking, Applications and Services, HEALTHCOM 2011, 2011(13), 15-20. https://doi.org/10.1109/HEALTH.2011.6026734

[3] Atzori, L., Lera, A., Morabito, G., Nitti, M. (2012). The social internet of things (Siot)- when social networks meet the internet of things: Concept architecture and networks characterization, computers", Computer Networks, Vol. 56, no. 16, pp. 3594-3608.

[4] Rahmani, A. M., Thanigaivelan, N. K., Gia, T. N., Granados, J., Negash, B., Liljeberg, P., \& Tenhunen, H. (2015). Smart e-Health Gateway: Bringing intelligence to Internet-of-Things based ubiquitous healthcare systems. 2015 12th Annual IEEE Consumer Communications and Networking Conference, CCNC 2015, (June), 826-834. https://doi.org/10.1109/CCNC.2015.7158084.

[5] Dujovne, D., Watteyne, T., Vilajosana, X., \& Thubert, P. (2014). 6T I Sch : D Deterministic Ip-E Nabled I Industrial I Nternet ( of T Hings ), (December), 36-41

[6] Drolet, A., Schwarz, N., \& Yoon, C. (2011). The ageing consumer: Perspectives from psychology and economics. The Aging Consumer: Perspectives from Psychology and Economics. New York: Taylor \& Francis. https://doi.org/10.4324/9780203852941

[7] Ehrenhard, M., Kijl, B., \& Nieuwenhuis, L. (2014). Market adoption barriers of multi-stakeholder technology: Smart homes for the ageing population. Technological Forecasting and Social Change, 89, 306-315.

[8] Corchado, J. M., Bajo, J., \& Abraham, A. (2008). GerAmi: Improving healthcare delivery in geriatric residences. IEEE Intelligent Systems, 23(2), 1925. https://doi.org/10.1109/MIS.2008.27.

[9] Christensen, K., Doblhammer, G., Rau, R., \& Vaupel, J. W. (2009). Ageing populations: the challenges ahead. The Lancet, 374(9696), 1196-1208. https://doi.org/10.1016/S01406736(09)61460

[10] Meinel, L., Findeisen, M., Hes, M., Apitzsch, A., \& Hirtz, G. (2014). Automated real-time surveillance for ambient assisted living using an 
omnidirectional camera. 2014 IEEE International Conference on Consumer Electronics (ICCE), 396-399. https://doi.org/10.1109/ICCE.2014.6776056

[11] Gobbens, R. J. J., Luijkx Katrien G., K. G., Wijnen-Sponselee, M. T., \& Schols, J. M. G. A. (2010). InSearch of an integral conceptual definition of frailty: Opinions of experts. Journal of the American Medical Directors Association, 11(5), 338-343. https://doi.org/10.1016/j.jamda.2009.09.015

[12] Kung, A., Jean/Bart, B.,(2010).Making ALL platforms a reality. In: de Ruyter B. et al. (eds) Ambient intelligence -Aml 2010.Lecture Notes in Computer Science, Vol6439. Springer, Berlin, Heidelberg.

[13] Rappaport, A. M. (2011). Living to 100: Growing Societal Impact And Challenges, 8(4).

[14] Misra, G., Kumar, V., Agarwal, A., \& Agarwal, K. (2016). Internet of Things (IoT) - A Technological Analysis and Survey on Vision, Concepts, Challenges, Innovation Directions, Technologies, and Applications (An Upcoming or Future Generation Computer Communication System Technology). American Journal of Electrical and Electronic Engineering, Vol. 4, 2016, Pages 23-32, 4(1), 23-32. https://doi.org/10.12691/AJEEE-4-1-4

[15] Instituto Nacional de Estatítica. (2014).Estatíticas Demográficas 2013. 73rd Edition. https://doi.org/0377-2284

[16] Kantoch, E., Augustyniak, P., Markiewicz, M., \& Prusak, D. (2014). Monitoring activities of daily living based on the wearable wireless body sensor network. 2014 36th Annual International Conference of the IEEE Engineering in Medicine and Biology Society, EMBC 2014, (August 2014), 586-589. https://doi.org/10.1109/EMBC.2014.6943659

[17] Oizumi, K., Kajiwara, H., \& Aratame, N. (2006). Facing up the problem of population ageing in developing countries. JICA, December. Tokyo: Research Group, Institute for International Cooperation (IFIC).
[18] Kale, S. S., \& Bhagwat, D. S. (2016). Highly Secured IoT Based Health Care System for Elderly People using Body Sensor Network based. IJIRSET, 5(10), 17796-17801. https://doi.org/10.15680/IJIRSET.2016.0510042

[19] Hsu, Y.-C., Tsai, C.-H., Kuo, Y.-M., \& Ya-Hui, B. (2016). Telecare Services for Elderly: Predictive Factors of Continued Use Intention. The Open $\begin{array}{llll}\text { Biomedical Engineering Journal, } & 10(1), & 82-90 .\end{array}$ https://doi.org/10.2174/1874120701610010082

[20] Storrs, C. (2016, April 21). People who are and lonely are at a greater risk of heart disease. Retrievedfrom:https://edition.cnn.com/2016/04/20/health/canloneliness-lead-to-heartdisease/index.html.

[21] Bottou, L. (2014). From machine learning to machine reasoning: An Essay. Machine Learning, 94(2), 133-149. https://doi.org/10.1007/s10994-013-5335 $\mathrm{x}$

[22] Ducatel, K., Bogdanowicz, M., Scapolo, F., Leijten, J., \& Burgelman, J.C. (2001). ISTAG Scenarios for Ambient Intelligence in 2010. User-Friendly Information Society, (October 2015), 58. https://doi.org/10.1111/jvh.12555

[23] Steele, R., Steele, R., Lo, A., Secombe, C., \& Kuen, Y. (2009). Elderly persons ' perception and acceptance of using wireless sensor networks to assist healthcare Elderly persons ' perception and acceptance of using wireless sensor networks to assist healthcare. International Journal of Informatics, 78(September 2009), 788-801. https://doi.org/10.1016/j.ijmedinf.2009.08.001

[24] Wagner, N., Hassanein, K., \& Head, M. (2010). Computer use by older adults: A multi-disciplinary review. Computers in Human Behavior, 26(5), 870-882. https://doi.org/10.1016/j.chb.2010.03.029 